\title{
Multiple View Image Rectification
}

\author{
Vincent Nozick \\ Gaspard Monge Institute, UMR 8049 \\ Paris-Est Marne-la-Vallee University, France \\ Email: vnozick@univ-mlv.fr
}

\begin{abstract}
This paper presents an extension of image rectification methods for an arbitrary number of views with aligned camera center. This technique can be used for stereoscopic rendering to enhance the perception comfort or for depth from stereo. In this paper, we first expose that epipolar geometry is not suited to solve this problem. Then we propose a non linear method that includes all the images in the rectification process. Our method only requires point correspondences between the views and can handle images with different resolutions. The tests show that the method is robust to noise and and to sparse point correspondences among the views.
\end{abstract}

\section{INTRODUCTION}

Stereo image rectification consists in the transformation of two images of the same scene such that for each pixel correspondence between the two views, the two pixels are aligned along the image horizontal axis. The main purpose of image rectification in computer vision is to optimize depth from stereo methods both in computational time and in robustness. A less known application concerns stereoscopic image visualization where the vertical parallax between any point correspondences should be minimized. The large majority of the proposed methods are dedicated to 2-views systems. In this paper, we propose an extension of image rectification to more than two views. Rectifying more than two views implies some constraints on the cameras' devices, especially the fact that the cameras' center should be aligned. Multiple view image rectification is essential for autostereoscopic camera setup and can have some applications for depth from stereo methods.

A basic approach consists of pairwise image rectifications. In this paper, we propose an alternative method that ensures a computation providing a globally optimized solution using simultaneously all the views rather than pair by pair. Our method can handle images with different resolution, orientation and focal length.

\section{IMAGE RECTIFICATION}

Image rectification methods have been known for long by photogrammetrists [1] and have been improved later by software approaches, like with Ayache and Hansen [2]. Most of this early methods involved to know the camera projection matrices. Then, this constraint has been relaxed with methods taking advantage of epipolar geometry to align the two images. The main issue of image rectification is that the rectified pair is not unique. Most of existing methods deal with how to find an image rectification that minimizes the image distortion.
Robert et al. [3] attempt to reduce the amount of distortion by finding the rectification transform that is closest to preserving orthogonality about the image centers. However, orthogonality is not an adequate criterion since even an Euclidean image rectification can involve a loss of orthogonality. Correcting this non-orthogonality might decrease the Euclidean property of the rectified images. Hartley [4] proposes a linear method that minimizes the horizontal parallax among the point correspondences used for the rectification. Loop and Zhang [5] decompose the rectification process into affine and projective components. Al-Zahrni et al. [6] propose a method that prevent the rectification process from a distortion on a selected common plane specified from 3 points correspondence on the two images. Gluckman et al. [7] propose a method to find the transformation that best preserves the sampling of the original stereo pair, i.e. each pixel in the unrectified image should map to a single pixel in the rectified image. Mallon and Whelan [8] optimize each transformation in order to minimize perspective distortions, such that the rectified images look like the original images as closely as possible. Monasse et al. [9] perform an image rectification by three successive rotations. Isgrò and Trucco [10] do not explicitly compute epipolar geometry but generate a rectifying pair of homographies that are are conform to the fundamental matrix form of a rectified image pair. Finally, Pollefeys et al. [11] propose a rectification method based on a reprojection onto a cylindrical surface instead of a plane in order to reach an optimal pixel distribution on the rectified images to avoid any pixel loss.

All these methods minimize an image distortion and thus are well suited for depth from stereo methods but not for stereoscopic rendering since they do not consider the camera transformation. In other words, there is no guarantee to obtain a pair of rectified images that corresponds or is close to an Euclidean camera setup. Moreover, most of these methods are based on epipolar geometry and hence cannot be directly extended to handle more than two views, as we will show in section V-B.

\section{RECTIFYING MORE THAN TWO VIEWS}

\section{A. Image rectification for stereoscopic rendering}

In recent years, stereoscopic systems advanced from stereoscopic to autostereoscopic displays. These devices can provide more than two views simultaneously, usually around ten views, and the users do not need to wear any specific glasses. To provide a comfortable stereoscopic rendering, a stereoscopic image should avoid vertical parallax between any 
correspondence points. This problem has been deeply studied (see Allison [12]) and can be corrected with an adequate image rectification if the cameras' center are aligned. This latter constraint makes possible to rectify an image with its right and left neighbors simultaneously. Finally, to reach orthostereoscopic rendering [13], i.e. the scene is perceived with the real geometric proportions, the image rectification should be Euclidean (i.e. metric). A quasi-Euclidean rectification where the respective camera focal lengths are coherent but only known up to a scale factor, provides a quasi-orthostereoscopic rendering that is also acceptable.

Zhou and Li [14] propose an image rectification dedicated to stereoscopic rendering based on epipolar geometry that provides good results, but is adapted only for two views. Fusiello and al. [15] and later Fusiello et al. [16] propose a non-linear method to rotate and zoom a pair of projective camera such that they fit to a pair of rectified camera, according to the epipolar constraints. This approach is specially well suited for stereoscopic rendering since this method provides quasiEuclidean image rectification. However, this method is hardly to be extended to multiple images rectification since it is based on an implicit epipolar relation between the two cameras. Our method is mainly related to this approach, especially concerning the quasi-Euclidean rectification.

\section{B. Rectifying more than two views}

Ayache and Hansen [2], Sun [17] and also An et al. [18] present some methods to perform an image rectification over three views. They combine a horizontal image rectification between a central image with a left image and vertical image rectification between the central image with a bottom image. This approach is designed to extend depth from stereo methods to three views, however this technique cannot be used for three aligned cameras.

Kang et al. [19] present an image rectification from multiple calibrated images. They adapt the images orientation and focal such that the cameras share a common image plane. Boutarel and Nozick [20] present a GPU image rectification that can support multiple images. This method requires the camera to be calibrated and performs a projection on a common image plane, followed by a back projection on a set of ideal rectified cameras. This method can handle slight misalignment of the camera center if the common image plane is chosen carefully since it minimizes the error around this common projection plane. The main drawback of these latter methods is the need of calibrated cameras when all the other methods can deal with only point correspondences.

\section{NOTATIONS}

In this paper, we will follow the same notation as in [21]. We indicate column vectors of $\mathbb{P}^{2}$ in bold lower-case letters such as $\mathbf{x}=(x, y, w)^{\top}$ and column vectors of $\mathbb{P}^{3}$ in bold upper case letters such as $\mathbf{X}$. Row vectors are transposed column vectors, such as $\mathbf{x}^{\top}$. The $y$ coordinate of a vector is denoted by $(\cdot)_{y}$. Matrices are denoted by upper case letters, such as $\mathrm{H}$.
A set of points is denoted by an italic upper case letter like $U$ and its cardinality by $|U|$.

\section{EPIPOLAR GEOMETRY AND MORE THAN TWO VIEWS}

Epipolar geometry is an usual support for stereo image rectification methods. Thus, it can also appear to be a good solution to rectify multiple images.

\section{A. Epipolar geometry}

The epipolar geometry describes the relations that exist between two images and can be described by the following equation:

$$
\mathbf{x}^{\prime \top} \mathbf{F} \mathbf{x}=0
$$

where $\mathbf{x}$ and $\mathbf{x}^{\prime}$ are homogeneous representation of corresponding points and the fundamental matrix $F$ is the algebraic representation of epipolar geometry. An epipole is the projection in one view of the camera center of the other view. Numerically, the epipoles $\mathbf{e}$ and $\mathbf{e}^{\prime}$ are the right and left null space of $\mathrm{F}$ and can be computed by solving the homogeneous equations:

$$
\mathrm{Fe}=\mathbf{0} \quad \mathrm{F}^{\top} \mathbf{e}^{\prime}=\mathbf{0}
$$

For more details about epipolar geometry, the reader can refer to [21].

\section{B. More than two views}

If we consider more than two views where the cameras' center are perfectly aligned, an extension of epipolar geometry is conceivable according to the fact that an image has a unique epipole whatever the other view. Indeed, if we consider $N$ aligned cameras, the projection of the $i^{t h}$ camera's center on the $j^{t h}$ image is equivalent to the intersection of the line passing throw all the camera center and the $j^{\text {th }}$ image plane. Since this epipole $\mathbf{e}_{i j}$ is constant $\forall i \in\{1 \cdots N\}_{i \neq j}$, then we note $\mathbf{e}_{i j}=\mathbf{e}_{j}$ and the equation (1) leads to $\mathbf{F}_{i j} \mathbf{e}_{j}=\mathbf{0}$, where $\mathrm{F}_{i j}$ is the fundamental matrix between the image $i$ and $j$. This equation can be extended in a least square form:

$$
\left[\begin{array}{c}
\mathrm{F}_{1 j} \\
\mathrm{~F}_{2 j} \\
\vdots \\
\mathrm{F}_{N j}
\end{array}\right] \mathbf{e}_{j}=\mathbf{0}
$$

Unfortunately, this approach is numerically unstable unless the cameras' center are perfectly aligned. For practical purposes, this condition is usually not perfectly satisfied. The alternative that consists of computing the average epipole between an image and all the others is also unstable since a misalignment of a camera center has strong consequences on the computed average epipole. Imagine the situation where an epipole is at infinity with one view and on a finite point with another. Computing the average position would have no meaning.

As a consequence, epipolar geometry appears to be incompatible with more than two views, neither by an overall process nor by pairwise computation. 


\section{OUTLINE OF OUR METHOD}

Image rectification process can be considered as a transformation on the images such that they share a common image plane. Let's consider a set of $N$ cameras with projection matrix $\mathrm{P}_{i}=\mathrm{K}_{i}\left[\mathrm{R}_{i} \mid \mathbf{C}_{i}\right]$. We want to find the corrected cameras $\mathrm{P}^{\prime}{ }_{i}=$ $\mathrm{K}_{i}^{\prime}{ }_{i}\left[\mathrm{R}_{i}^{\prime} \mid \mathbf{C}_{i}^{\prime}{ }_{i}\right]$ such that all the cameras' focal plane become coplanar. This transformation can be expressed by a rotation around the optical center and an update of the focal length. Here, we do not have to consider the cameras' position. Thus, the relation between the initial and the corrected projection matrix can be defined as an homography $\mathrm{H}_{i}$ :

$$
\mathrm{H}_{i}=\mathrm{K}_{i}^{\prime} \mathrm{R}_{i}^{\prime}\left(\mathrm{K}_{i} \mathrm{R}_{i}\right)^{-1}=\mathrm{K}_{i}^{\prime} \mathrm{R}_{i}{ }_{i} \mathrm{R}_{i}^{-1} \mathrm{~K}_{i}^{-1}
$$

In this equation, $\widehat{\mathrm{R}}_{i}=\mathrm{R}^{\prime}{ }_{i} \mathrm{R}_{i}^{-1}$ corresponds to the rotation applied to $\mathrm{P}_{i}$ to have the same the orientation as $\mathrm{P}^{\prime}{ }_{i}$. Thus, equation (3) can be rewritten as follows:

$$
\mathrm{H}_{i}=\mathrm{K}_{i}^{\prime} \widehat{\mathrm{R}}_{i} \mathrm{~K}_{i}^{-1}
$$

The problem becomes how to find $\widehat{\mathrm{R}}_{i}$ and $\mathrm{K}^{\prime}{ }_{i}$ such that $\mathrm{H}_{i}$ rectify the images. Given some point correspondences between each view, we want to find $\mathrm{H}_{i}$ such that the transformed point correspondences are horizontally aligned.

This method is not related to epipolar geometry and hence can be extended for an arbitrary number of views. Moreover, it does not involve any prior knowledge about the cameras' projection matrices and requires only point correspondences between the views. Finally, this method is well suited for stereoscopic rendering since the operations guaranty a quasiEuclidean rectification.

\section{Multiple View image ReCTIFICATION}

Consider a set of point correspondences $\left\{\mathbf{x}_{U}^{k}\right\}$ where $U^{k}$ denotes the set of cameras involved by the $k^{t h}$ correspondence and where $\forall k,\left|U^{k}\right| \geq 2$ (i.e. each correspondence relates at least two views).

Let $\mathrm{K}_{i}^{\prime}$ and $\widehat{\mathrm{R}}_{i}$ the rectifying internal parameters and rotation for each camera, then the rectified point correspondences should be horizontally aligned:

$$
\left(\mathrm{H}_{i} \mathbf{x}_{i}^{k}\right)_{y}=y^{k}, \forall i \in U^{k}
$$

where $y^{k}$ represents the vertical coordinate of the rectified point $k$ on each view. In practice, the $y^{k}$ can be set as the average $y$-coordinate of the $k^{t h}$ rectified points since the rectified point correspondences should have the same $y$-coordinate:

$$
y^{k}=\frac{\sum_{i \in U^{k}}\left(\mathrm{H}_{i} \mathbf{x}_{i}^{k}\right)_{y}}{\left|U^{k}\right|}
$$

Hence, the homographies $\mathrm{H}_{i}$ should satisfy:

$$
\left(\mathrm{H}_{i} \mathbf{x}_{i}^{k}\right)_{y}-y^{k}=0
$$

We propose to find $\mathrm{H}_{i}$ with a non-linear process where each $\mathrm{K}_{i}^{\prime}$ and $\widehat{\mathrm{R}}_{i}$ are optimized to satisfy equation (5) by minimizing the residual error $e$ over all rectified point correspondences:

$$
e=\sum_{k} \frac{\sum_{i \in U^{k}}\left|\left(\mathrm{H}_{i} \mathbf{x}_{i}^{k}\right)_{y}-y^{k}\right|}{\left|U^{k}\right|}
$$

The error computation of each correspondence $k$ is normalized by $\left|U^{k}\right|$ since the correspondance points may not involve all the views. We perform a bundle adjustment on $\mathrm{K}_{i}^{\prime}$ and $\widehat{\mathrm{R}}_{i}$ from each view using Levenberg-Marquartd method.

To simplify the computation process, we reduce the number of parameters of $\mathrm{K}_{i}^{\prime}$ by specifying a zero-skew, a unit aspect ratio and a principal point centered on the image. The only remaining parameter is the focal length. Hence, the cameras' internal parameter matrix is defined as follows:

$$
\mathrm{K}_{i}=\left[\begin{array}{ccc}
f_{i} & 0 & w_{i} / 2 \\
0 & f_{i} & h_{i} / 2 \\
0 & 0 & 1
\end{array}\right]
$$

where $w_{i}$ and $h_{i}$ are respectively the width and height image resolution of the $i^{t h}$ view. An usual initial value for the focal length $f_{i}$ is $f_{i}^{0}=\sqrt{w_{i}^{2}+h_{i}^{2}}$. As suggested in [22] and [16], it is numerically recommended to represent the focal length by a variable ranging in $[-1,1]$ using zero as an initial value for the non-linear computation. It is common to expect $f$ to have a value roughly ranging in $\left[f_{i}^{0} / 3, f_{i}^{0} \times 3\right]$, thus $f_{i}$ is represented by a value $\alpha_{i}=\log _{3}\left(f_{i} / f_{i}^{0}\right)$. Hence, starting the non-linear process with the default focal length induces an initial value of $\alpha_{i}$ set to zero. Then, during the non-linear process, the current focal length is computed by:

$$
f_{i}=f_{i}^{0} 3^{\alpha_{i}}
$$

Each rotation matrix $\widehat{\mathrm{R}}_{i}$ is defined by three Euler angles $\theta_{i}^{x}, \theta_{i}^{y}$ and $\theta_{i}^{z}$ such that $\widehat{\mathrm{R}}_{i}=\mathrm{R}_{\theta_{i}^{z}} \mathrm{R}_{\theta_{i}^{y}} \mathrm{R}_{\theta_{i}^{x}}$. For each view, the unknowns are the focal length represented by $\alpha_{i}$ and the three Euler angles $\theta_{i}^{x}, \theta_{i}^{y}$ and $\theta_{i}^{z}$ which make a total of four unknowns per camera.

All the variables $\left(\theta_{i}^{x}, \theta_{i}^{y}, \theta_{i}^{z}, \alpha_{i}\right)$ are set to zero at the beginning of the procedure, meaning no orientation change on the cameras and the default focal length. To avoid a free rotation of all the images around the cameras' base-line, we force the angle $\theta^{x}$ to be zero for one of the views during the minimization process. We also let the focal length of this camera constant since the focal length of the cameras are defined up to scale.

As specified in equation (6), the point correspondences do not have to cover all the views. The only constraint is that each view should be directly or indirectly linked to all the others. Finally, our method can handle multiple view image rectification but is also very well suited for a two image rectification.

\section{DIFFERENT IMAGE RESOLUTION}

In our method presented in section VII, we consider that all the input images have the same resolution. If it is not the case, the method should be adapted to overcome some computational errors. More specifically, a change in the focal length may lead to a misalignment for two images with different resolutions if the origin of the pixel coordinates is not centered. Indeed, the focal length variations provide centered transformations, but the image coordinates are not subject to this scale, as described in equation (4). Figure 1 shows that 


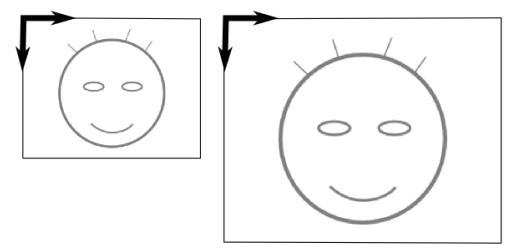

(a)

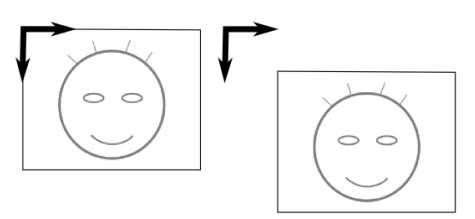

(b)
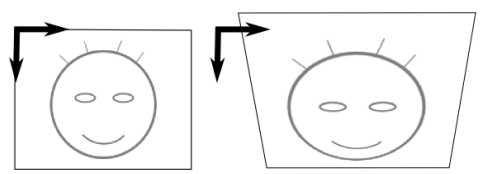

(c)

Fig. 1. (a) Input images with different resolution. (b) A centered variation of the focal length of the second image. The scale is good but not the alignment. (c) A better alignment cost is found by a combining a rotation around the camera baseline and a centered variation of the focal length on the second image, however this image rectification is not optimal.

even with an adequate update of the focal length, the images are not aligned. This misalignment may lead to a rotation along the image baseline that will distort the image and degrade the image rectification. This problem does not occur if the pixel coordinates are centered, as depicted in Figure 2.

Therefore, our method should be modified such that the data is centered. Let $\mathrm{H}_{i}^{c}$ be the homography that centers the pixels of the $i^{\text {st }}$ image, defined as:

$$
\mathrm{H}_{i}^{c}=\left[\begin{array}{ccc}
1 & 0 & -w_{i} / 2 \\
0 & 1 & -h_{i} / 2 \\
0 & 0 & 1
\end{array}\right]
$$

Equation (4) becomes:

$$
\mathrm{H}_{i}=\mathrm{H}_{\text {out }}^{c-1} \mathrm{~K}_{i}^{\prime} \widehat{\mathrm{R}}_{i} \mathrm{~K}_{i}^{-1} \mathrm{H}_{i}^{c}
$$

Where the matrix $\mathrm{H}_{\text {out }}^{c}$ is a centering homography corresponding to the output image format that specifies a common pixel coordinates origin for all the images. In practice, $\mathrm{H}_{\text {out }}^{c}$ can be the centering homography of the smallest image. Moreover, the intrinsic parameters matrices should not include the origin of the image, since the data is already centered. These matrices become:

$$
\mathrm{K}_{i}=\left[\begin{array}{ccc}
f_{i} & 0 & 0 \\
0 & f_{i} & 0 \\
0 & 0 & 1
\end{array}\right]
$$

Finally, the non-linear process can find the best parameters to rectify the images. It is not required for the input views to have neither the same image resolution nor the same focal length. The bundle adjustment process is summarized by the minimization function described in Algorithm 1.

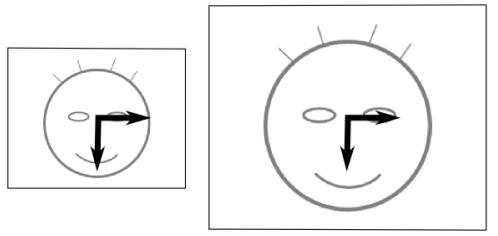

(a)
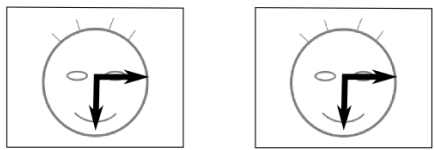

(b)

Fig. 2. (a) Input images with different resolution. (b) A centered variation of the focal length of the second image. Both the scale and the alignment are satisfying.

If all the input images have the same resolution, Algorithm 1 can be simplified by replacing the equation of line 3 with equation (7) and the line 6 with equation (4). However using Algorithm 1 provides very good results whatever the image resolution and does not alter the numerical performance even if the images have the same resolution.

\section{RESUlts}

We implemented our method in C++ using LevenbergMarquardt method implemented from [21]. Note that this implementation of Levenberg-Marquardt is the easiest form compared to its standard description for more complex problems. To make our method automatic, we use SURF detector [23] to find correspondence points between the views. The outliers are detected using RANSAC applied on the epipolar geometry between each consecutive pair of images. Since this RANSAC procedure is not performed on every images simultaneously, the issue about epipolar geometry for multiple view presented in section V-A does not hold. Figure 5 depicts an example of our method applied on four images.

We tested our method on synthetic data consisting in a set of aligned projective cameras with controlled focal length, image resolution and orientation. A bunch of random $3 \mathrm{D}$ points are projected on each camera to get point correspondences. We performed this procedure on five $(800 \times 600)$ virtual cameras and 50 point correspondences with various camera settings:

1) identical cameras.

2) different orientations, same focal length.

3) same orientation, different focal lengths.

4) different orientations and focal length.

where "different" means a $10 \%$ variation between each view on the concerned parameter. In this situation, even if we have the ground truth camera calibration parameters, there is no quantitative measure of the distortion that reflects the quality of the image rectification. Therefore we compute the average of the $y$-axis disparity between the average $y^{k}$ and the rectified points among each correspondence $k$. The procedure is repeated with an isotropic Gaussian noise on the correspondence 


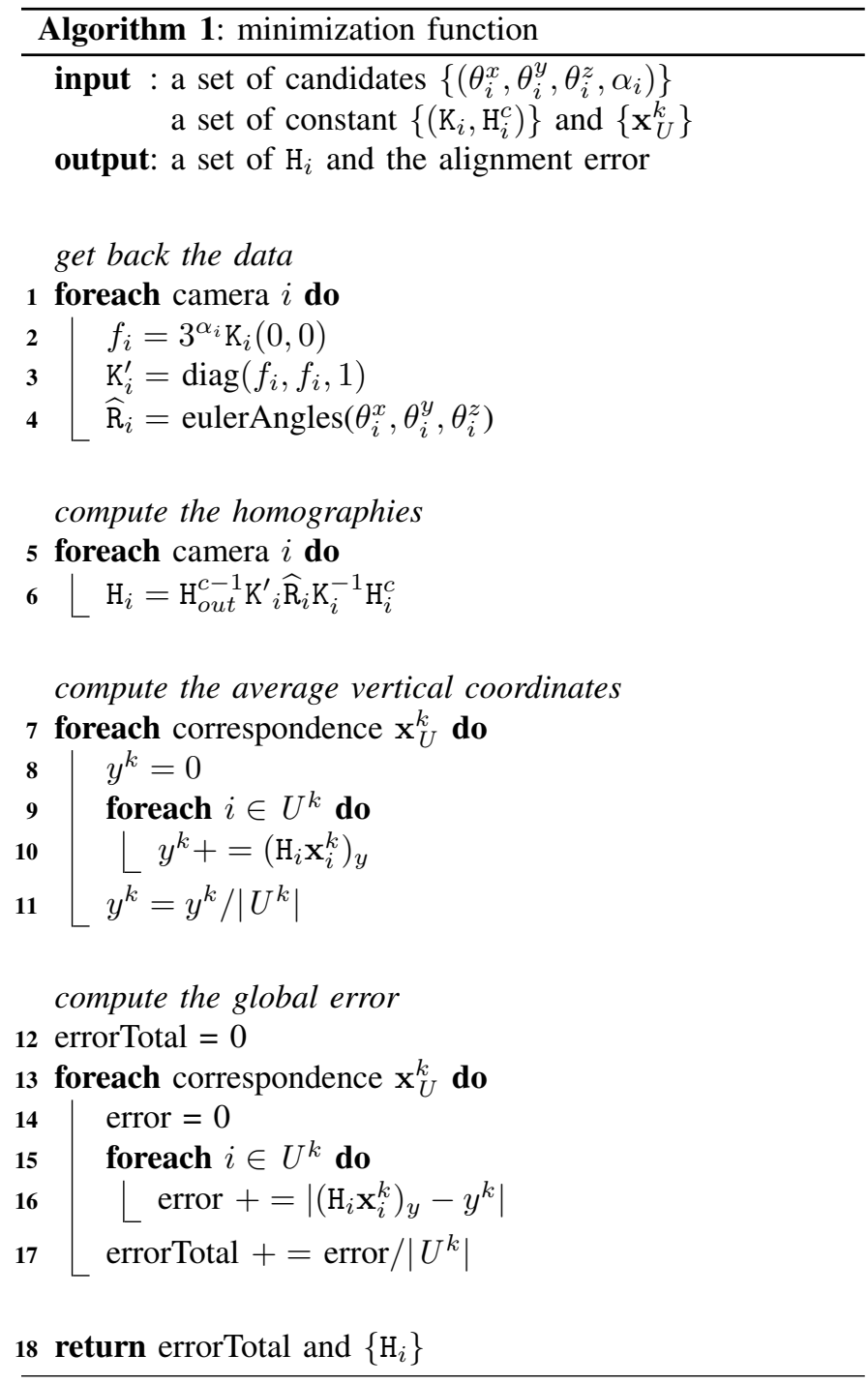

points with various amplitudes. These results are summarized in Figure 3 and show that the proposed method is robust to Gaussian noise. The tests also show that the method is a bit more efficient to correct orientation than focal length.

The second part of our tests concerns the point correspondences density. We removed some point correspondences from the full data set such that some correspondences do not relate all the views. The results are presented in Figure 4 and clearly show that our method still provides very good results, even when a large part of the point correspondences are missing.

Finally, our tests show that our method succeed to perform a quasi-Euclidean image rectification and hence can be exploited for a quasi-orthostereoscopic rendering. The computation of the matrices $\mathrm{H}_{i}$ should be performed only once at the beginning of the video acquisition process and the video stream rectification can be computed online on the GPU as shown in [20].

\begin{tabular}{|c|c|c|c|}
\hline data set & zero-noise & $\begin{array}{c}\text { Gaussian noise } \\
\text { ampl.: 2 pixels }\end{array}$ & $\begin{array}{c}\text { Gaussian noise } \\
\text { ampl.: 5 pixels }\end{array}$ \\
\hline 1 & 0.000 & 0.540 & 1.363 \\
2 & 0.064 & 0.553 & 1.360 \\
3 & 0.130 & 0.568 & 1.328 \\
4 & 0.108 & 0.557 & 1.369 \\
\hline
\end{tabular}

Fig. 3. Average disparity (in pixel) on the $y$-axis between 50 point correspondences with five $(800 \times 600)$ images applied on various data sets defined in section IX. The data is corrupted by an isotropic Gaussian noise with standard deviation $\sigma=0.4$ under various amplitudes.

\begin{tabular}{|c|c|c|c|c|}
\hline data set & full data & $90 \%$ data & $60 \%$ data & $40 \%$ data \\
\hline 1 & 0.000 & 0.000 & 0.000 & 0.000 \\
2 & 0.064 & 0.112 & 1.006 & 1.996 \\
3 & 0.130 & 0.066 & 0.071 & 0.067 \\
4 & 0.108 & 0.036 & 0.056 & 1.164 \\
\hline
\end{tabular}

Fig. 4. Average disparity (in pixel) with sparse point correspondences on the $y$-axis. The tests are performed with different correspondences density by randomly removing some correspondences. The full data contains 50 point correspondences relating the five $(800 \times 600)$ images. These tests are applied on various data sets defined in section IX.

\section{COnClusion}

This paper presents a image rectification method that can handle more than two images. This method is not related to epipolar geometry and hence can be extended for an arbitrary number of views. Like other techniques that rectify multiple images, the only constraint on the cameras is that their center should be aligned. Our method does not involve any prior knowledge about the camera projection matrices and requires only point correspondences between the views. Moreover, the point correspondences between the views do not have to concern all the images simultaneously. Finally, the method supports input images with different image resolutions.

This method is well suited to remove vertical parallax for stereoscopic rendering without any damage on the perspective perception since the operations performed during the rectification process guaranty a quasi-Euclidean rectification. This quasi-Euclidean rectification ensures a quasi-orthostereoscopic rendering where the scene is perceived with the real geometric proportions. To our knowledge, it is the only method that provides quasi-orthostereoscopic images without full camera calibration.

Our method has been validated by several tests concerning both the robustness with inaccurate point correspondence and sparse point correspondences over all views.

As a future work, we plan to extend this method for a camera calibration purpose as an alternative to the classical bundle adjustment [24].

\section{REFERENCES}

[1] C. Slama, Manual of Photogrammetry, 4th ed. American Society of Photogrammetry, 1980. 1

[2] N. Ayache and C. Hansen, "Rectification of images for binocular and trinocular stereovision," in 9th International Conference on Pattern Recognition, ser. ICPR '88, vol. 1. Rome, Italy: IEEE Computer Society, November 1988, pp. 11-16. 1, 2 

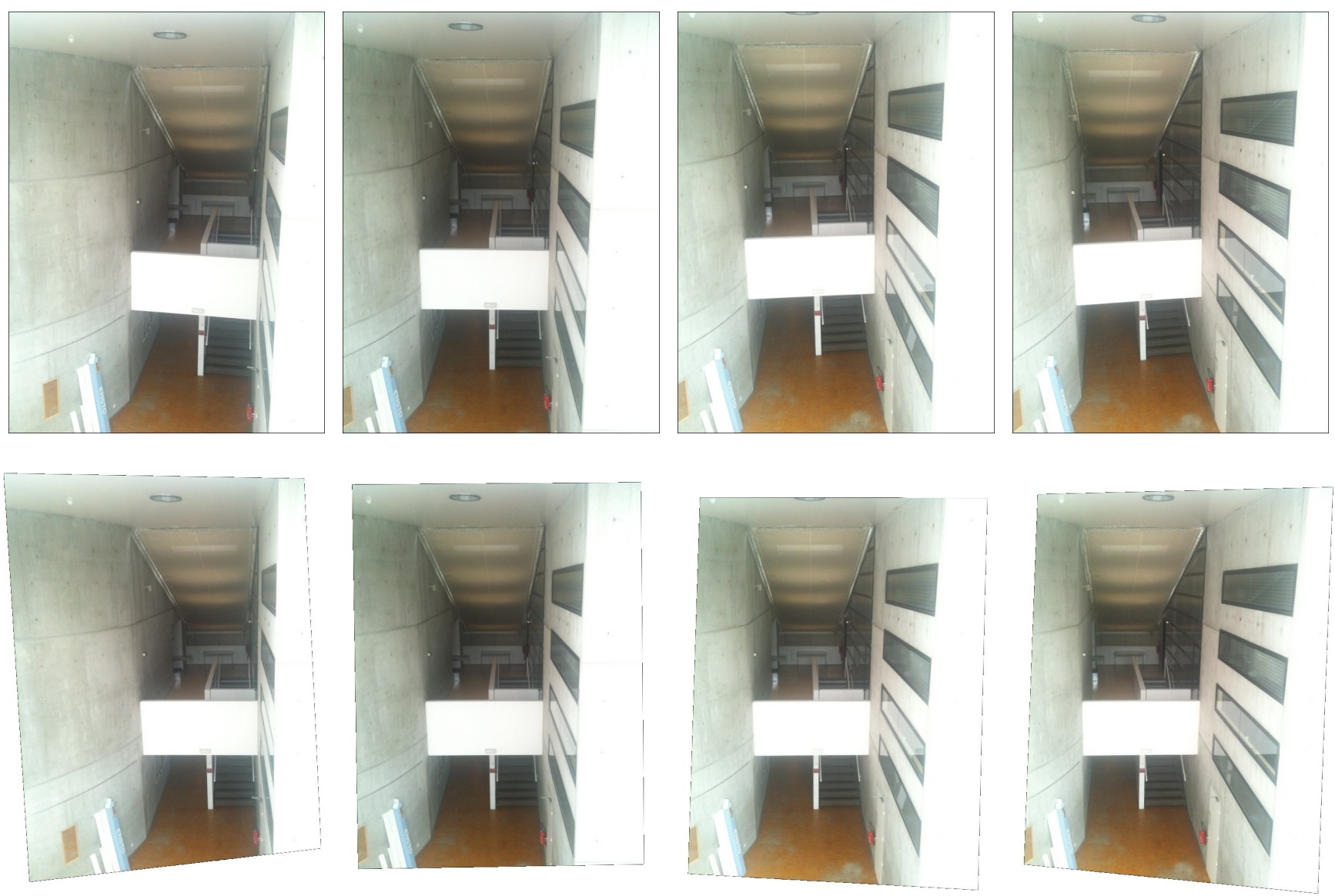

Fig. 5. Multiple image rectification : (Up) four input images. (Down) the four rectified images.

[3] L. Robert, C. Zeller, O. Faugeras, and M. Hébert, "Applications of nonmetric vision to some visually guided robotics tasks," INRIA, Tech. Rep. RR-2584, June 1995. 1

[4] R. I. Hartley, "Theory and practice of projective rectification," International Journal of Computer Vision, vol. 35, no. 2, pp. 115-127, 1999. 1

[5] C. Loop and Z. Zhang, "Computing rectifying homographies for stereo vision," Computer Vision and Pattern Recognition, vol. 1, p. 1125, 1999. 1

[6] A. Al-Zahrani, S. S. Ipson, and J. G. B. Haigh, "Applications of a direct algorithm for the rectification of uncalibrated images," Inf. Sci. Inf. Comput. Sci., vol. 160, pp. 53-71, March 2004. 1

[7] J. Gluckman and S. K. Nayar, "Rectifying transformations that minimize resampling effects," Computer Vision and Pattern Recognition, IEEE Computer Society Conference on, vol. 1, p. 111, 2001. 1

[8] J. Mallon and P. F. Whelan, "Projective rectification from the fundamental matrix," Image Vision Comput., vol. 23, pp. 643-650, July 2005. 1

[9] P. Monasse, J.-M. Morel, and Z. Tang, "Three-step image rectification," in Proceedings of BMVC, September 2010, pp. 89.1-10. 1

[10] F. Isgrò and E. Trucco, "Projective rectification without epipolar geometry," Computer Vision and Pattern Recognition, IEEE Computer Society Conference on, vol. 1, pp. 94-99, 1999. 1

[11] M. Pollefeys, R. Koch, and L. V. Gool, "A simple and efficient rectification method for general motion," IEEE International Conference on Computer Vision, vol. 1, p. 496, 1999. 1

[12] R. Allison, "An analysis of the influence of vertical disparities arising in toed-in stereoscopic cameras," Journal of Imaging Science and Technology, vol. 51, pp. 317-327, 2007. 2

[13] M. T. M. Lambooij, W. A. IJsselsteijn, and I. Heynderickx, "Visual discomfort in stereoscopic displays: a review," Stereoscopic Displays and Virtual Reality Systems XIV, vol. 6490, no. 1, 2007. 2
[14] J. Zhou and B. Li, "Image rectification for stereoscopic visualization," JOSA A, vol. 25, no. 11 , pp. 2721-2733, November 2008. 2

[15] A. Fusiello, E. Trucco, and A. Verri, "A compact algorithm for rectification of stereo pairs," Machine Vision and Applications, vol. 12, no. 1, pp. $16-22,1999.2$

[16] A. Fusiello, E. Trucco, A. Verri, and R. Verri, "Quasi-Euclidean epipolar rectification," International Conference on Pattern Recognition, pp. 1-4, 2008. 2, 3

[17] C. Sun, "Uncalibrated three-view image rectification," Image and Vision Computing, vol. 21, pp. 259-269, 2003. 2

[18] L. An, Y. Jia, J. Wang, X. Zhang, and M. Li, "An efficient rectification method for trinocular stereovision," in Proceedings of the 17th International Conference on Pattern Recognition, ser. ICPR '04, vol. 4. Washington, DC, USA: IEEE Computer Society, 2004, pp. 56-59. 2

[19] Y. Kang, C. Lee, and Y. Ho, "An efficient rectification algorithm for multi-view images in parallel camera array," in 3DTV08, 2008, pp. 6164. 2

[20] F. Boutarel and V. Nozick, "Epipolar rectification for autostereoscopic camera setup," in EAM Mechatronics 2010, Yokohama, Japan, November 2010, pp. 133-136. 2, 5

[21] R. I. Hartley and A. Zisserman, Multiple View Geometry in Computer Vision, 2nd ed. Cambridge University Press, ISBN: 0521540518, 2004. 2,4

[22] G. Medioni and S. B. Kang, Emerging Topics in Computer Vision. Upper Saddle River, NJ, USA: Prentice Hall PTR, 2004. 3

[23] C. Evans, "Notes on the opensurf library," University of Bristol, Tech. Rep. CSTR-09-001, January 2009. 4

[24] B. Triggs, P. McLauchlan, R. I. Hartley, and A. Fitzgibbon, "Bundle adjustment - a modern synthesis," Vision Algorithms: Theory and Practice, pp. 153-177, 2000. 5 